\title{
Theoretical Developments of the LaMET Approach to Parton Physics
}

\author{
Yong Zhao*a,b \\ ${ }^{a}$ Center for Theoretical Physics, Massachusetts Institute of Technology, Cambridge, \\ Massachusetts 02139, USA \\ ${ }^{b}$ Physics Department, Brookhaven National Laboratory Bldg. 510A, Upton, NY 11973, USA \\ E-mail: yzhao@bnl.gov
}

\begin{abstract}
The large-moment effective theory (LaMET) is a systematic approach to extract light-cone parton physics from equal-time matrix elements that are calculable in lattice QCD. Recent years have seen rapid developments of the LaMET approach which have been applied to various lattice calculations and led to much promising progress in this field. In this work, we will discuss the formalism of LaMET and its extension to parton distributions for the transverse structures, which are essential steps in the road map to obtaining the three-dimensional tomography of the hadrons from lattice QCD.
\end{abstract}

37th International Symposium on Lattice Field Theory - Lattice2019

16-22 June 2019

Wuhan, China

${ }^{*}$ Speaker. 


\section{Introduction}

One of the most important goals of hadron physics nowadays is the precise understanding of three-dimensional hadronic structures. These structures are described by a class of parton distributions that can be interpreted as the density of partons in the infinite momentum frame (IMF). For example, the collinear parton distribution functions (PDF) were first introduced to describe the number density of partons in the longitudinal momentum space, which also include spin-dependent structures such as helicity and transversity. In addition to the collinear PDFs, genearlized parton distributions (GPDs) give the color charge density distribution in the transverse position space, and transverse momentum dependent (TMD) PDFs measure the transverse momentum of partons. Moreover, all the above distributions can be projected from a phase-space Wigner distribution or generalized TMD (GTMD), which also contains more abundant structures such as the parton orbital angular momentum distributions in the hadron.

On the experimental side, the global analysis of deep inelastic scattering (DIS) and hadronhadron collision data in the past few decades have led to remarkable quantification of the collinear PDFs, such as the unpolarized and helicity valence quark PDFs. Nevertheless, other distributions such as spin-dependent gluon and sea quark PDFs, as well as the GPDs and TMDPDFs, still have quite significant uncertainties due to the limitation of currently available experiments. The planned Electron-Ion Colliders in the US [1], China [2] and Europe [3], with unprecedented luminosity, polarization and kinematic coverage, are aimed at completing the missing pieces on this jigsaw puzzle.

On the theoretical side, it would be highly desirable if first principle methods such as lattice QCD can provide an independent determination of these parton distributions. Comparing to the cost and complexity of building more powerful and versatile colliders, the lattice calculation of hadronic structures requires tremendous supercomupting resources which are attainable in the forseable future. From a more practical angle, lattice QCD can provide the necessary input to constrain the models used in the global fitting of the longitudinal and transverse parton distributions, thus improving the experimental extraction of these observables. For example, a recent global fit of the transversity quark PDF $\delta q(x)$ which was combined with the lattice result of the tensor charge $\delta q=\int d x \delta q(x)$ has seen significant reduction of uncertainties [4].

Neverthless, it is extremely difficult to directly calculate light-cone correlations on a Euclidean lattice due to their explicit time dependence. A few years ago, Ji proposed the large-momentum effective theory (LaMET) [5, 6] to directly extract the $x$-dependence of the PDFs on the lattice, which has seen remarkable progress in its application to lattice calculations. The exploratory study of this method was first carried out for the isovector quark PDFs in the proton [7, 8, 9], which has been systematically improved over the past years and obtained promising results for a precise determination of PDFs from lattice QCD [10, 11, 12, 13, 14]. Meanwhile, the extension of LaMET to the lattice calculations of gluon and singlet quark PDFs, as well as GPDs, are also being explored on the lattice $[15,16]$. More recently, efforts are being made to generalize this method to the TMDPDFs [17, 18, 19, 20, 21, 22, 23], which has been used to calculate the nonperturbative Collins-Soper evolution kernel on the lattice [19, 24, 25].

In this work, we review the formalism of LaMET in Sec. 2, and show how this approach can be used to calculate all PDFs on the lattice in Sec. 3. Finally, we will discuss the extension of 
the LaMET method to the transverse hadronic structures, i.e., GPDs and TMDPDFs, in Sec. 4. In particular, we will show how the nonperturbative Collins-Soper evolution kernel for the TMDPDFs can be extracted from lattice QCD, and show some preliminary lattice results for this observable. We conclude in Sec. 5 .

\section{Large-Momentum Effective Theory}

LaMET is a systematic approach to calculate light-cone parton physics from lattice QCD [5, 6]. The key idea is to approximate a light-cone observable by a highly boosted static quasi observable that is calculable on the lattice, and then extract the former from the latter through a factorization formula that can be quantified in perturbative QCD [26, 27, 28].

Take the calculation of quark PDF as example. The light-cone quark PDF is defined as

$$
q(x, \mu) \equiv \int \frac{d \xi^{-}}{4 \pi} e^{-i x P^{+} \xi^{-}}\left\langle P\left|\bar{\psi}\left(\xi^{-}\right) \gamma^{+} U\left(\xi^{-}, 0\right) \psi(0)\right| P\right\rangle,
$$

where $x$ is the momentum fraction, the nucleon momentum $P^{\mu}=\left(P^{0}, 0,0, P^{z}\right), \xi^{ \pm}=(t \pm z) / \sqrt{2}$ are the light-cone coordinates, and the Wilson line is

$$
U\left(\xi^{-}, 0\right)=P \exp \left(-i g \int_{0}^{\xi^{-}} d \eta^{-} A^{+}\left(\eta^{-}\right)\right)
$$

$\mu$ is the renormalization scale as the PDF is usually defined in the $\overline{\mathrm{MS}}$ scheme. In light-cone quantization with $A^{+}=0$, the $\overline{\mathrm{MS}}$ definition has an interpretation as a parton number density in the longitudinal momentum space.

Due to the explicit time dependence in the light-cone coordinate $\xi^{-}$, the quark PDF cannot be directly obtained from the lattice. According to LaMET, one can approximate it by the "quasiPDF" which is defined from an equal-time correlator in the hadron state,

$$
\tilde{q}\left(x, P^{z}, \mu\right) \equiv \int_{-\infty}^{\infty} \frac{d z}{4 \pi} e^{i x P^{z} z}\langle P|\bar{\psi}(z) \Gamma U(z, 0) \psi(0)| P\rangle,
$$

where $\Gamma=\gamma^{z}$ or $\gamma^{t}, z^{\mu}=z \hat{z}^{\mu}, \hat{z}^{\mu}=(0,0,0,1)$, and the Wilson line is

$$
U(z, 0)=P \exp \left(-i g \int_{0}^{z} d z^{\prime} A^{z}\left(z^{\prime}\right)\right) .
$$

Since the light-cone PDF is defined to be boost invariant along the longitudinal direction, it does not depend on the external momentum of the hadron. The momentum fraction $x$ of the partons in the PDF is confined to $[-1,1]$, with negative $x$ correpsonding to the antiquarks. This is because the light-cone coordinate correpsonds to the IMF where quarks or gluons moving in the backward direction are suppressed. In contrast, the quasi-PDF defined from the equal-time correlator is not boost invariant, and depends dynamically on the hadron momentum $P^{z}$. Moreover, the quasi-PDF has support $x \in(-\infty, \infty)$, because in the finite momentum frame the quarks and gluons can escape the hadron and move in backward directions.

By calculating the quasi-PDF in a large-momentum hadron state, it is equivalent to boosting the equal-time correlator towards the light-cone. Therefore, one might naively think that the lightcone PDF can be obtained by taking the IMF limit by extrapolating to $P^{z} \rightarrow \infty$. Unfortunately, 
due to noncommutativity between the $P^{z} \rightarrow \infty$ and ultra-violet (UV) regularization in quantum field theory, the IMF of the quasi-PDF cannot be directly taken, which is similar to the situation of heavy quark effective theory (HQET) where the heavy quark mass $M_{Q} \rightarrow \infty$ limit cannot be exchanged with the UV regularization.

Nevertheless, we can perform a large $P^{z}$ expansion near infinity instead, just like that in HQET. This expansion is guaranteed by the factorization formula $[26,27,28]$,

$$
\tilde{q}\left(x, P^{z}, \mu\right)=\int_{-1}^{1} \frac{d y}{|y|} C\left(\frac{x}{y}, \frac{\mu}{|y| P^{z}}\right) q(y, \mu)+\mathscr{O}\left(\frac{M^{2}}{P_{z}^{2}}, \frac{\Lambda_{\mathrm{QCD}}^{2}}{x^{2} P_{z}^{2}}\right),
$$

where the leading power contribution can be factorized into the convolution of a matching coefficient $C$ and the light-cone PDF. The power corrections originate from the target-mass and highertwist effects, which are suppresed by the large hadron momentum, except that the higher-twist correction is suppressed by the large parton momentum $x P^{z}$.

Based on the factorization formula in Eq. (2.4), one can establish a systematic procedure to calculate the PDF from lattice QCD:

1) Lattice simulation of the bare quasi-PDF. This requires a lattice calculation of the equaltime correlator at large hadron momentum. With currently available lattice ensembles, one can perform lattice calculation with $P^{z}=2 \sim 3 M_{N}$ for the proton and $P^{z}=3 \sim 5 M_{\pi}$ for the pion [11, $13,14,29,30]$.

2) Renormalization of the quasi-PDF on the lattice. To make sure that after matching corrections the final result has a controllable continuum limit, it is necessary to perform a nonperturbative renormalization of the quasi-PDF on the lattice, so that the matching is between renormalized quantities. To ensure a well defined renormalization procedure on the lattice, a multiplicative renormalizability is required for the quasi-PDF, which has been proven in coordinate space $[31,32,33]$. As a result, a nonperturbative renormalization of the quasi-PDF can be performed in the coordinate space. In recent years, several renormalization schemes have been proposed. One way is to determine the mass correction $\delta m$ nonperturbatively from the static quark-antiquark potential $[34,35,36]$, and then use lattice perturbation theory to match to the $\overline{\mathrm{MS}}$ scheme in the continuum $[35,26]$. However, it is still not clear how to match $\delta m$ from the nonperturbative lattice scheme to $\overline{\mathrm{MS}}$. Another way that has been popularly implemented is the regualrization independent momentum subtraction (RI/MOM) scheme [37, 38, 39, 40], which can be umambiguously matched to the $\overline{\mathrm{MS}}$ scheme $[37,38]$, except that at large $|z|$ the perturbation theory may need to be revisited. It has also been propsed to renormalize the equal-time correlator by its matrix element at zero hadron momentum, which is referred to as the "reduced pseudo Ioffe-time disribution" [41, 42]. This method has the advantage of being renormalization group invariant and has less systematic uncertainties due to the correlated errors. Nevertheless, at large $|z|$, the nonperturbative highertwist corrections in the renormalization factor also make the matching unreliable. After all, novel approaches to renormalize the quasi-PDF is still favored to reduce the systematic corrections from this procedure.

3) Power corrections. There are two types of power corrections. One is the familiar target mass corrections [43] in the twist-two contributions of the quasi-PDF, and has been derived to all orders for the quark case in Ref. [8]. The other is the genuine higher-twist corrections, whose explicit form at twist-four has been derived in Ref. [8], but still needs to be calculated nonperturbatively from 
lattice QCD. For current calculations, one can perform all the other power and matching corrections first, and then extrapolate to the $P^{z} \rightarrow \infty$ limit, assuming that logarithmic $P^{z}$ dependence has been sufficiently canceled by the matching coefficient.

4) Perturbative matching. As a key part of the LaMET approach, the perutrbative matching is necessary for us to obtain the final light-cone PDF. The matching coefficients have been derived at one-loop order in the transverse momentum cutoff regularization [26], $\overline{\mathrm{MS}}$ scheme [44, 28], and the RI/MOM scheme [38, 45, 14] for the unpolarized, helicity and transversity quark quasi-PDFs. A two-loop matching is necessary to understand the convergence of perturbation series.

\section{Gluon and Singlet-quark PDFs from Lattice QCD}

Ever since LaMET was proposed, it has been explored on the lattice calculation of non-singlet quark PDFs [7, 9]. The nonperturbative renormalization was implemented in more recent calculations [39, 40], and reached remarkable agreement with the global fits for the unpolarized and helicity cases $[12,11,13]$ at physical pion mass. Notably, for the transversity case, the lattice results have smaller uncertainties than experimental fits [10, 14], which can be of great phenomenological significance in the near future. Meanwhile, lattice calculations of the pion distribution amplitude [36, 46] and PDF [29, 30] have also been made.

A natural extension of LaMET is from the flavor non-singlet case to the singlet case. Since the singlet quark PDF mixes with the gluon PDF, it would also require the calculation of the latter at the same time.

The gluon quasi-PDF can be defined in the following way,

$$
\tilde{g}\left(x, P^{z}\right) \equiv N \int \frac{d z}{2 \pi x P^{z}} e^{i x P^{z} z}\left\langle P\left|\tilde{O}_{g}(z)\right| P\right\rangle=N \int \frac{d z}{2 \pi x P^{z}} e^{i x P^{z} z}\left\langle P\left|\mathscr{P}_{\mu \nu} F^{n_{1} \mu}(z) W(z, 0) F^{n_{2} v}(0)\right| P\right\rangle,
$$

where $W(z, 0)$ is a Wilson line in the adjoint representation, $F^{n \mu}=n_{\rho} F^{\rho \mu}$ and $n_{1}^{\mu}, n_{2}^{\mu}$ are either $\hat{z}^{\mu}$ or $\hat{v}^{\mu}=(1,0,0,0)$. The projector $\mathscr{P}$

$$
\mathscr{P}_{\mu v}=g_{\mu v} \text { or } g_{\mu v}-\hat{v}_{\mu} \hat{v}_{v} / \hat{v}^{2}-\hat{z}_{\mu} \hat{z}_{v} / \hat{z}^{2} .
$$

The normalization factor $N=\left(P^{z} / P^{t}\right)^{\delta n_{1} v+\delta n_{2} v}$.

It has also been proven that the gluon Wilson line operators can be multiplicatively renormalized in coordinate space $[47,48]$, up to mixings with equation-of-motion operators that contribute to a contact term [47]. Moreover, using operator product expansion, the factorization formula for the gluon and quark-singlet PDF has also been rigorously derived [49]:

$$
\begin{gathered}
\tilde{q}_{i}\left(x, P_{z}, \mu\right)=\int_{-1}^{1} \frac{d y}{|y|}\left[\sum_{j} C_{q_{i} q_{j}}\left(\frac{x}{y}, \frac{\mu}{|y| P^{z}}\right) q_{j}(y, \mu)+C_{q g}\left(\frac{x}{y}, \frac{\mu}{|y| P^{z}}\right) g(y, \mu)\right]+\mathscr{O}\left(\frac{M^{2}}{P_{z}^{2}}, \frac{\Lambda_{\mathrm{QCD}}^{2}}{x^{2} P_{z}^{2}}\right), \\
\tilde{g}\left(x, P_{z}, \mu\right)=\int_{-1}^{1} \frac{d y}{|y|}\left[\sum_{j} C_{g q}\left(\frac{x}{y}, \frac{\mu}{|y| P^{z}}\right) q_{j}(y, \mu)+C_{g g}\left(\frac{x}{y}, \frac{\mu}{|y| P^{z}}\right) g(y, \mu)\right]+\mathscr{O}\left(\frac{M^{2}}{P_{z}^{2}}, \frac{\Lambda_{\mathrm{QCD}}^{2}}{x^{2} P_{z}^{2}}\right),
\end{gathered}
$$


where $i, j$ runs over both quark and anti-quark flavors. The matching coefficients have been derived at one-loop order in the transverse momentum cutoff, $\overline{\mathrm{MS}}$ and RI/MOM schemes [50, 51, 49].

An exploratory study focusing on the calculation of gluon quasi-PDF has been made [15], which has not included the lattice renormalization. The implementation of the RI/MOM for the gluon quasi-PDF, however, should be studied for its systematic uncertanties.

\section{Transverse Hadron Structures from Lattice QCD}

The three-dimensional hadron structures can be described by the generalized parton distributions (GPDs) and transverse momentum dependent (TMD) PDFs. The former gives the color charge densities in the tranverse coordinate space in the IMF, whereas the latter is the density distribution in the transverse momentum space. Both can be derived from a phase space Wigner or GTMD, which contains more abundant structures such as the spin-orbtial correlation of the partons.

The principles of LaMET can be applied to these observables, which have also seen rapid progress in recent years.

GPDs. The GPDs are collinear distributions defined from the off-forward matrix elements of hadrons. For the non-singlet quark case, the GPD is defined from the light-cone correlator,

$$
F(x, \xi, t)=\int \frac{d z^{-}}{4 \pi} e^{-i x z^{-} \bar{P}^{+}}\left\langle P_{2}\left|\bar{\psi}\left(\frac{z}{2}\right) \gamma^{+} U\left(\frac{z}{2},-\frac{z}{2}\right) \psi\left(-\frac{z}{2}\right)\right| P_{1}\right\rangle,
$$

where

$$
\bar{P}=\frac{P_{1}+P_{2}}{2}, \quad \Delta=P_{2}-P_{1}, \quad P_{1}^{2}=P_{2}^{2}=M^{2}, \quad t=\Delta^{2},
$$

and the skewness parameter is

$$
\xi \equiv-\frac{\Delta^{+}}{2 \bar{P}^{+}} .
$$

At zero skewness $\xi=0$, the GPD can be interpreted as number density of partons in the Fourier conjugate space of $\Delta[52,53]$.

Similarly, the quasi-GPD can be defined from the equal-time correlator as

$$
\tilde{F}(x, \tilde{\xi}, t)=\int \frac{d z}{4 \pi} e^{x z \bar{P}^{z}}\left\langle P_{2}\left|\bar{\psi}\left(\frac{z}{2}\right) \Gamma U\left(\frac{z}{2},-\frac{z}{2}\right) \psi\left(-\frac{z}{2}\right)\right| P_{1}\right\rangle,
$$

where $\Gamma=\gamma^{z}$ or $\gamma^{t}$, and the quasi skewness

$$
\tilde{\xi} \equiv-\frac{\Delta^{z}}{2 \bar{P}^{z}}=\xi+\mathscr{O}\left(\frac{M^{2}}{P_{z}^{2}}\right) .
$$

The factorization formula for the quasi-GPD has also been proved using operator production expansion [54], which take the following two equivalent forms:

$$
\begin{aligned}
\widetilde{F}\left(x, \xi, t, P^{z}, \mu\right) & =\int_{-1}^{1} \frac{d y}{|\xi|} \bar{C}\left(\frac{x}{\xi}, \frac{y}{\xi}, \frac{\mu}{\xi P^{z}}\right) F(y, \xi, t, \mu)+\mathscr{O}\left(\frac{M^{2}}{P_{z}^{2}}, \frac{t}{P_{z}^{2}}, \frac{\Lambda_{\mathrm{QCD}}^{2}}{x^{2} P_{z}^{2}}\right), \\
& =\int_{-1}^{1} \frac{d y}{|y|} C\left(\frac{x}{y}, \frac{\xi}{y}, \frac{\mu}{y P^{z}}\right) F(y, \xi, t, \mu)+\mathscr{O}\left(\frac{M^{2}}{P_{z}^{2}}, \frac{t}{P_{z}^{2}}, \frac{\Lambda_{\mathrm{QCD}}^{2}}{x^{2} P_{z}^{2}}\right),
\end{aligned}
$$


where the two matching coefficients in Eqs. (4.6) and (4.7) are related to each other by

$$
C\left(\frac{x}{y}, \frac{\xi}{y}, \frac{\mu}{y P^{z}}\right)=\left|\frac{y}{\xi}\right| \bar{C}\left(\frac{x}{\xi}, \frac{y}{\xi}, \frac{\mu}{\xi P^{z}}\right) .
$$

Since the quasi-GPDs are defined from the same Wilson line operators as the quasi-PDFs, their renormalization can be done in exactly the same way, except that the matching coefficient needs to be recalculated for the bare matrix element. The one-loop matching coeffcients have been derived in Ref. [54]. An exploratory calculation of the pion non-singlet quark GPD has been performed using the RI/MOM renormalization [16].

TMDs TMDPDFs (and TMD fragmentation functions) can be measured in semi-inclusive deep inelastic scattering, Drell-Yan like processes and dihadron production of electron-positron annihilation. Take the Drell-Yan process as example, for the production of a color-singlet final state partile(s) with center of mass enery $Q$, if one measures the transverse momentum $q_{T}$, the differential cross section satisfies a factorization theorem when $q_{T} \ll Q[55,56,57,58,59,60,61,62,63,64]$,

$$
\begin{aligned}
\frac{d \sigma}{d Q d Y d^{2} q_{T}}= & \sum_{i j} H_{i j}(Q, \mu) \int d^{2} b_{T} e^{\vec{b}_{T} \cdot \vec{q}_{T}} B_{i}\left(x_{a}, \vec{b}_{T}, \mu, \frac{\zeta_{a}}{v^{2}}\right) B_{j}\left(x_{b}, \vec{b}_{T}, \mu, \frac{\zeta_{b}}{v^{2}}\right) \\
& \times S_{i j}\left(b_{T}, \mu, v\right)\left[1+\mathscr{O}\left(\frac{q_{T}^{2}}{Q^{2}}, \frac{\Lambda_{\mathrm{QCD}}^{2}}{Q^{2}}\right)\right],
\end{aligned}
$$

where $H$ is a hard function calculable in perturbation theory, $B_{i, j}$ are the collinear beam functions describing the initial state partons, whereas $S_{i, j}$ is a soft function that captures the radiation of soft gluons in the entire phase space. $i, j$ are flavor indices. Due to the overlap of the soft and collinear degrees of freedom, both the beam and soft functions suffer from the so-called rapidity divergence (or light-cone divergence) that occurs when one tries to separate them, which needs an additional regulator ${ }^{1}$. After renormalization, both the beam and soft functions depend on the renormalization scale $\mu$ and rapidity scale $v$. $\zeta_{a}$ and $\zeta_{b}$ are called the Collins-Soper scales and are related to the energy of the incoming partons, $\zeta_{a} \zeta_{b}=Q^{4}$.

Due to rapidity regularization scheme dependence, it is usually more convenient to absorb the soft function into the beam function and define the so-called physical TMDPDFs that are universal in TMD processes,

$$
f_{i}^{\mathrm{TMD}}\left(x, \vec{b}_{T}, \mu, \zeta\right)=B_{i}\left(x, \vec{b}_{T}, \mu, \frac{\zeta}{v^{2}}\right) \sqrt{S^{i}\left(b_{T}, \mu, v\right)},
$$

so that the TMD factorization formula can be expressed in a more intuitive form

$$
\begin{aligned}
f_{i}^{\mathrm{TMD}}\left(x, \vec{b}_{T}, \mu, \zeta\right)= & \sum_{i, j} H_{i j}(Q, \mu) \int d^{2} b_{T} e^{\overrightarrow{i b}_{T} \cdot \vec{q}_{T}} \\
& \times f_{i}^{\mathrm{TMD}}\left(x_{a}, \vec{b}_{T}, \mu, \zeta_{a}\right) f_{j}^{\mathrm{TMD}}\left(x_{b}, \vec{b}_{T}, \mu, \zeta_{b}\right)\left[1+\mathscr{O}\left(\frac{q_{T}^{2}}{Q^{2}}, \frac{\Lambda_{\mathrm{QCD}}^{2}}{Q^{2}}\right)\right] .
\end{aligned}
$$

When $b_{T} \ll \Lambda_{\mathrm{QCD}}^{-1}$, the soft function is perturbative, while the beam function can be matched onto the collinear PDFs with logarithms of $\left(b_{T} \mu\right)$. Instead, when $b_{T} \sim \Lambda_{\mathrm{QCD}}^{-1}$, both the beam and

\footnotetext{
${ }^{1}$ For a review of different rapidity regularization schemes, see Ref. [20].
} 
soft functions are intrinsically nonperturbative and can only be determined through global fitting or first principle calculations.

To fit the TMDPDFs using Eq. (4.11), we must know how to evolve the TMDPDFs to different renormalization and Collins-Soper scales, which are described by two independent evolution equations,

$$
\begin{gathered}
\mu \frac{d \ln f_{i}^{\mathrm{TMD}}}{d \mu}=\gamma_{\mu}^{i}(\mu, \zeta), \\
\frac{1}{2} \zeta \frac{d \ln f_{i}^{\mathrm{TMD}}}{d \zeta}=\gamma_{\zeta}^{i}\left(\mu, b_{T}\right),
\end{gathered}
$$

where the anomalous dimension $\gamma_{\zeta}^{i}\left(\mu, b_{T}\right)$ is also called the Collins-Soper kernel. The two anomalous dimensions are related by the cusp anomalous dimension,

$$
\frac{d \gamma_{\zeta}^{i}\left(\mu, b_{T}\right)}{d \ln \mu}=2 \frac{d \gamma_{\mu}^{i}(\mu, \zeta)}{d \ln \zeta}=-2 \Gamma_{\text {cusp }}^{i}\left[\alpha_{s}(\mu)\right],
$$

which guarantees the analyticity of the TMDPDF in the $\mu-\zeta$ plane and allows us to uniquely evolve the TMDPDFs from initial scales $\mu_{0}, \zeta_{0}$ to arbitary scales,

$$
\begin{aligned}
& f_{i}^{\mathrm{TMD}}\left(x, \vec{b}_{T}, \mu, \zeta\right)=f_{i}^{\mathrm{TMD}}\left(x, \vec{b}_{T}, \mu_{0}, \zeta_{0}\right) \\
& \times \exp \left[\int_{\mu_{0}}^{\mu} \frac{d \mu^{\prime}}{\mu^{\prime}} \gamma_{\mu}^{i}\left(\mu^{\prime}, \zeta_{0}\right)\right] \exp \left[\frac{1}{2} \gamma_{\zeta}^{i}\left(\mu, b_{T}\right) \ln \frac{\zeta}{\zeta_{0}}\right] .
\end{aligned}
$$

Apparently, when $b_{T} \sim \Lambda_{\mathrm{QCD}}^{-1}$, the Collins-Soper kernel is nonperturbative, which poses extra challenge for the global fit of the TMDPDFs.

To approximate the physical TMDPDF according to LaMET, we start by constructing the quasi beam function $\tilde{B}_{q}$ and soft factor $\tilde{S}_{q}\left(b_{T}, a, L\right)$ on the lattice $[18,19,20]$, i.e.,

$\tilde{f}_{q}^{\mathrm{TMD}}\left(x, \vec{b}_{T}, \mu, P^{z}\right)=\lim _{L \rightarrow \infty} \int \frac{d b^{z}}{2 \pi} e^{i b^{z}\left(x P^{z}\right)} \tilde{Z}^{\prime}\left(b^{z}, \mu, \tilde{\mu}\right) \tilde{Z}_{\mathrm{UV}}\left(b^{z}, \mu, a\right) \tilde{B}_{q}\left(b^{z}, \vec{b}_{T}, a, L, P^{z}\right) / \sqrt{\tilde{S}_{q}\left(b_{T}, a, L\right)}$,

where $L$ is the size of the staple, which is limited by the finite volume of the lattice. $\tilde{Z}_{\mathrm{UV}}\left(b^{z}, \mu, a\right)$ renormalizes the overall UV divergences on the lattice, and $\tilde{Z}^{\prime}\left(b^{z}, \mu, \tilde{\mu}\right)$ converts the renormalized quasi TMDPDF into the $\overline{\mathrm{MS}}$ scheme. $\tilde{\mu}$ are scales introduced in lattice renormalization, and their dependence are canceled by the conversion factor. In the end, and infinite $L$ limit must be taken to obtain the quasi TMDPDF.

The light-cone beam function is defined from the matrix element of a staple-shaped Wilson line operator

$$
B_{q}\left(x, \vec{b}_{T}, \varepsilon, \tau\right)=\int \frac{d b^{-}}{2 \pi} e^{-i\left(x P^{+}\right) b^{-}}\left\langle P\left|\bar{q}\left(b^{\mu}\right) W\left(b^{\mu}\right) \frac{\gamma^{+}}{2} W_{T}\left(-\infty^{-} ; \vec{b}_{T}, \overrightarrow{0}_{T}\right) W^{\dagger}(0) q(0)\right|_{\tau} \mid P\right\rangle,
$$

where the configuration of the Wilson lines are shown in Fig. 1a. Here $\tau$ is the rapidity regulator.

Correpsondingly, the quasi beam function can be constructed with a staple-shaped Wilson line extending in the $z$ direction, as shown in Fig. 1b,

$$
\tilde{B}_{q}\left(x, \vec{b}_{T}, a, L, P^{z}\right)=\int \frac{d b^{z}}{2 \pi} e^{i b^{z}\left(x P^{z}\right)}\left\langle P\left|\bar{q}\left(b^{\mu}\right) W_{\hat{z}}\left(b^{\mu} ; L-b^{z}\right) \frac{\Gamma}{2} W_{T}\left(L \hat{z} ; \vec{b}_{T}, \overrightarrow{0}_{T}\right) W_{\hat{z}}^{\dagger}(0) q(0)\right| P\right\rangle .
$$




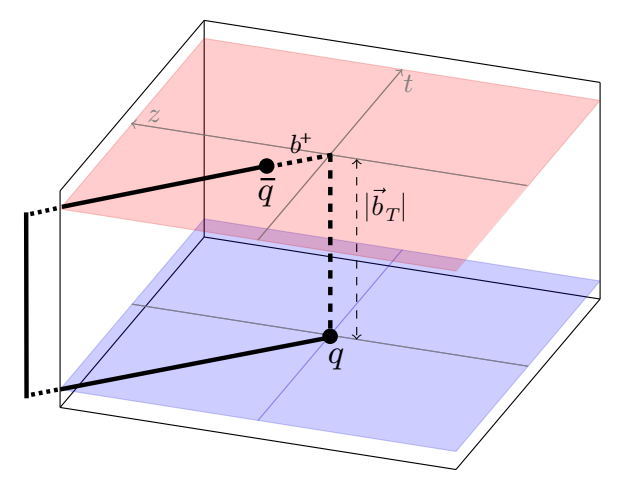

(a)

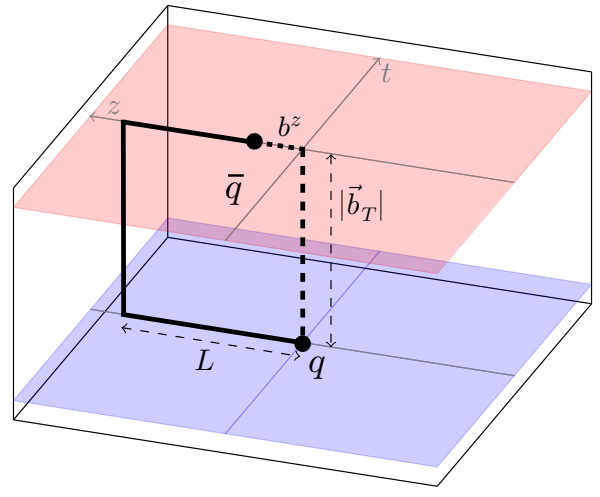

(b)

Figure 1: Wilson line configurations for the beam and quasi beam functions.

The quasi beam function is related to the beam function through a Lorentz boost in the $z$ direction, so one expects that the LaMET matching would work out for them, though in practice the rapidity regulator could spoil this relation. Nevertheless, one can still hope that after including the soft factor that cancels scheme dependence of the rapidity regulator, a factorization formula could still exist for the quasi and physical TMDPDFs.

The soft factor in TMD factorization theorem is defined from the vacuum matrix element of a Wilson loop that invovles two light-cone directions, as shown in Fig. 2a,

$$
S_{q}\left(b_{T}, \varepsilon, \tau\right)=\frac{1}{N_{c}}\left\langle\left. 0\left|\operatorname{Tr}\left[S_{n}^{\dagger}\left(\vec{b}_{T}\right) S_{\bar{n}}\left(\vec{b}_{T}\right) S_{T}\left(-\infty \bar{n} ; \vec{b}_{T}, \overrightarrow{0}_{T}\right) S_{\bar{n}}^{\dagger}\left(\overrightarrow{0}_{T}\right) S_{n}\left(\overrightarrow{0}_{T}\right) S_{T}^{\dagger}\left(-\infty n ; \vec{b}_{T}, \overrightarrow{0}_{T}\right)\right]\right|\right|_{\tau} \mid 0\right\rangle,
$$

where $N_{c}=3$, and $n$ and $\bar{n}$ are the collinear and anti-collinear light-cone directions.

A naive construction of the quasi soft factor would be a Wilson loop defined in the equal-time plane, as shown in Fig. 2b,

$\tilde{S}_{q}\left(b_{T}, a, L\right)=\frac{1}{N_{c}}\left\langle 0\left|\operatorname{Tr}\left[S_{\hat{z}}^{\dagger}\left(\vec{b}_{T} ; L\right) S_{-\hat{z}}\left(\vec{b}_{T} ; L\right) S_{T}\left(L \hat{z} ; \vec{b}_{T}, \overrightarrow{0}_{T}\right) S_{-\hat{z}}^{\dagger}\left(\overrightarrow{0}_{T} ; L\right) S_{n}\left(\overrightarrow{0}_{T} ; L\right) S_{T}^{\dagger}\left(-L \hat{z} ; \vec{b}_{T}, \overrightarrow{0}_{T}\right)\right]\right| 0\right\rangle$.

As one can see, the naive quasi soft factor cannot be boosted to the light-cone soft factor. Actually, all the other constructions of quasi soft factor from equal-time Wilson lines cannot be boosted to the configuration in Fig. 2a. This suggests that a perturbative matching relation between the quasi and physical TMDPDFs cannot be established like the quasi-PDF case.

Nevertheless, since the boost argument in LaMET only fails for the soft sector which does not depend on the external hadron state, it indicates that the mismatch between the physical and quasi TMDPDFs only depends, if nonperturbatively, on $b_{T}$. Therefore, it was argued in Ref. [20] that they satisfy the following relation (for the non-singlet case),

$$
\begin{aligned}
\tilde{f}_{\mathrm{ns}}^{\mathrm{TMD}}\left(x, \vec{b}_{T}, \mu, P^{z}\right)= & C_{\mathrm{ns}}^{\mathrm{TMD}}\left(\mu, x P^{z}\right) g_{q}^{S}\left(b_{T}, \mu\right) \exp \left[\frac{1}{2} \gamma_{\zeta}^{q}\left(\mu, b_{T}\right) \ln \frac{\left(2 x P^{z}\right)^{2}}{\zeta}\right] \\
& \times f_{\mathrm{ns}}^{\mathrm{TMD}}\left(x, \vec{b}_{T}, \mu, \zeta\right)+\mathscr{O}\left(\frac{b_{T}}{L}, \frac{1}{b_{T} P^{z}}, \frac{1}{P^{z} L}\right) .
\end{aligned}
$$




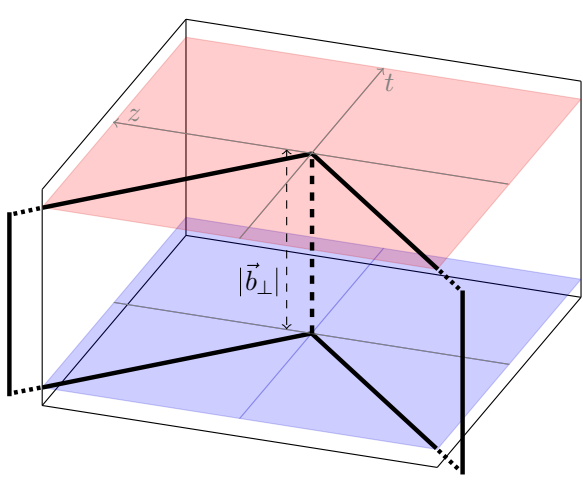

(a)

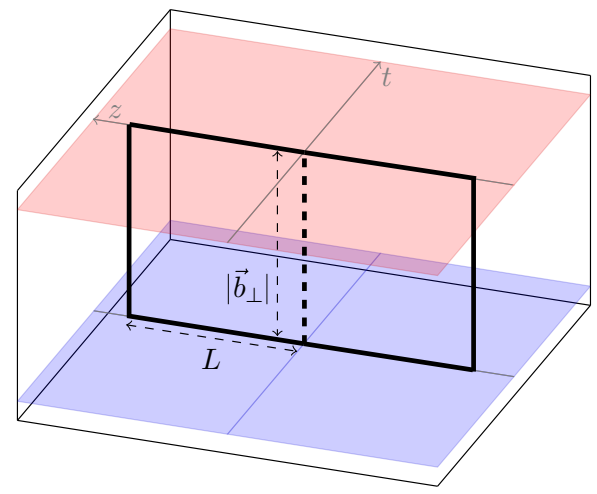

(b)

Figure 2: Wilson line configurations for the soft and quasi soft functions.

Here the exponential is required to cancel the $\zeta$ dependence in the physical TMDPDF, and $g_{q}^{S}\left(b_{T}, \mu\right)$ can be a nonperturbative function due to the failure of the boost argument for the soft sector. The perturbative matching coefficient $C_{\mathrm{ns}}^{\mathrm{TMD}}\left(\mu, x P^{z}\right)$ can be calculated by identifying $g_{q}^{S}\left(b_{T}, \mu\right)$ at each loop order.

Very recently, it is proposed that the soft factor in TMD factorization theorem can be calculated using HQET on the lattice or extracted from the form factor of a current-current correlator [22, 23]. Instead of starting from Wilson line operators, these methods utilize the Wilson-line-heavyquark equivalence or large momentum factorization of the lattice matrix element to obtain the soft factor regulated by off-lightcone Wilson lines [65]. This proposal would guarantee a perturbative matching between the quasi and physical TMDPDFs, and has the potential to predict the Drell-Yan cross section from the lattice [23].

Irregardless of the calculation of the soft sector, it was soon pointed out that the quasi-TMDPDF can be used to form ratios at different nucleon momenta to extract the nonperturbative CollinsSoper kernel [19]. The master formula is

$$
\begin{aligned}
\gamma_{\zeta}^{q}\left(\mu, b_{T}\right) & =\frac{1}{\ln \left(P_{1}^{z} / P_{2}^{z}\right)} \ln \frac{C_{\mathrm{ns}}^{\mathrm{TMD}}\left(\mu, x P_{2}^{z}\right) \tilde{f}_{\mathrm{ns}}^{\mathrm{TMD}}\left(x, \vec{b}_{T}, \mu, P_{1}^{z}\right)}{C_{\mathrm{ns}}^{\mathrm{TMD}}\left(\mu, x P_{1}^{z}\right) \tilde{f}_{\mathrm{ns}}^{\mathrm{TMD}}\left(x, \vec{b}_{T}, \mu, P_{2}^{z}\right)} \\
& =\frac{1}{\ln \left(P_{1}^{z} / P_{2}^{z}\right)} \ln \frac{C_{\mathrm{ns}}^{\mathrm{TMD}}\left(\mu, x P_{2}^{z}\right) \int d b^{z} e^{i b^{z} x P_{1}^{z}} \tilde{Z}^{\prime}\left(b^{z}, \mu, \tilde{\mu}\right) \tilde{Z}_{\mathrm{UV}}\left(b^{z}, \tilde{\mu}, a\right) \tilde{B}_{\mathrm{ns}}\left(b^{z}, \vec{b}_{T}, a, L, P_{1}^{z}\right)}{C_{\mathrm{ns}}^{\mathrm{TMD}}\left(\mu, x P_{2}^{z}\right) \int d b^{z} e^{i b^{z} x P_{2}^{z}} \tilde{Z}^{\prime}\left(b^{z}, \mu, \tilde{\mu}\right) \tilde{Z}_{\mathrm{UV}}\left(b^{z}, \tilde{\mu}, a\right) \tilde{B}_{\mathrm{ns}}\left(b^{z}, \vec{b}_{T}, a, L, P_{2}^{z}\right)} .
\end{aligned}
$$

Since the nonperturbative function $g_{q}^{S}\left(b_{T}, \mu\right)$ and the quasi soft factor $\tilde{S}_{q}\left(b_{T}, a, L\right)$ do not depend on $b^{z}$ or $P^{z}$, they cancel out in the ratio of the quasi-TMDPDFs, and we are only left to calculate the quasi beam function and renormalize it nonperturbatively on the lattice. The final result should be independent of the choice of $x$ and $P^{z}$, which indicates that there must be a stablized region which we can exploit to constrain the corresponding systematics. Finally, since the CollinsSoper kernel is a property associated with the operator itself, it does not depend on the external hadron state, and one can perform a calculation with the simplest hadron on the lattice, i.e. pions, even with a heavy valence pion mass. The idea of forming such ratios to access information of the $x$-moments of TMDPDFs has already been implemented in Refs. [66, 34, 67, 68, 69, 70]. 
An exploratory calculation of the Collins-Soper kernel using the above method is already underway. As an important step, the nonperturbative renormalization of staple-shaped Wilson line operators on the lattice has been studied using the RI/MOM scheme [24], and the conversion factors that convert the RI/MOM quasi beam function to the $\overline{\mathrm{MS}}$ scheme has also been calculated at oneloop order for $b^{z}=0$ [71] and $b^{z} \neq 0$ [21] cases. On a fine quenched lattice with $a=0.06 \mathrm{fm}$ and heavy pion mass $M_{\pi}=1.2 \mathrm{GeV}$, the Collins-Soper kernel has been extracted from the quasi beam functions at $P^{z}=1.2$ and $1.9 \mathrm{GeV}$ [25], as shown in Fig. 3.

In Fig. 3, the lattice results are compared to the Collins-Soper kernel from perturbation theory predicitons with effective quark flavor number equal to zero. As one can see, the solid and dashed curves become singular at $b_{T} \sim 0.25 \mathrm{fm}$ due to the Landau pole in the perturbation theory, which indicates that the latter is no longer reliable. In the small $b_{T}$ region where perturbation theory is reliable, the exploratory lattice results still differ from the expected curve qualitatively. Nevertheless, since there are also signifcant systematics in this region such as power corrections, this comparison can be improved in the future with larger hadron momentum and lattice volume. Moreover, the fact that these lattice results are of the same order magnitude as the perturabtive predictions gives promising sign that this method can provide robust predictions in the future.

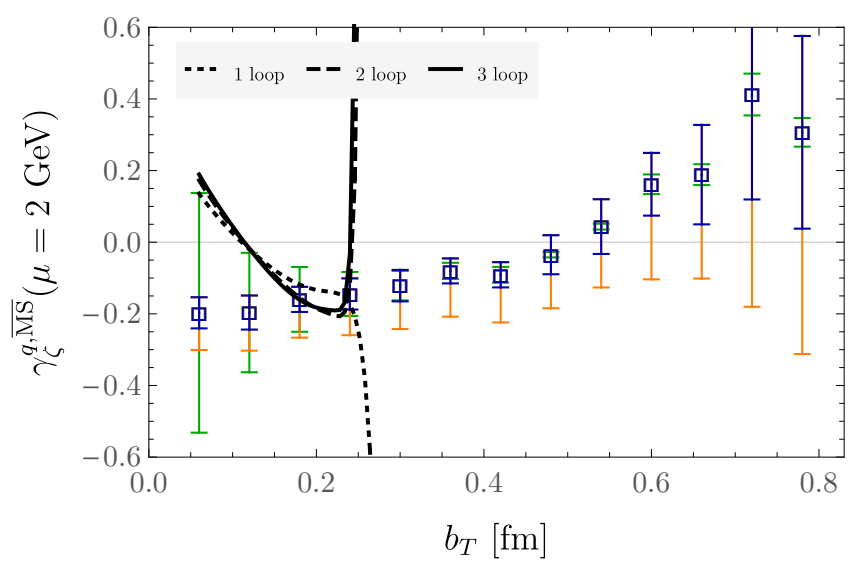

Figure 3: Collins-Soper kernel from an exploratory calculation. Blue error bars present statistical uncertainties and systematics from lattice renormalization; green error bars estimate the power corrections given by $1 /\left(P^{z} b_{T}\right)$; orange error bar estimates the uncertainty in the choice of $x$ values for the final results.

\section{Summary}

We have discussed the principles of LaMET, which allows for a lattice calculation of parton physics by utilizing the Lorentz boost picture of the parton model. For the lattice calculation of collinear PDFs, a systematic procedure has already been set up and applied to the calculation of isovector quark PDFs, and tremendous improvement has been made over the past years. The extension of this approach to the calculation of gluon and singlet quark PDFs, as well as the PGDs, is also straightforward, and the factorization formulas with one-loop perturbative mathcing coefficients have already been understood, and the relevant lattice calculations have just started. 
Finally, much progress has been made towards the lattice calculation of TMDPDFs with LaMET. The construction of the quasi beam and soft functions have been studied in perturbation theory, and there have been proposals to completely determine the soft factor from the lattice. An immediate application of the current findings is the lattice calculation of the nonperturbative Collins-Soper kernel, which is an important input for the global fitting of TMDPDFs from experiments. Preliminary lattice results have shown encouraging signs that lattice QCD can achieve a robust prediction of the Collins-Soper kernel in the future. With TMDPDFs and GPDs being calculable in the future, one can expect that the Wigner distributions are also within reach of LaMET, which would help us fully undrstand other issues such as the parton orbital angular momentum and spin-orbit correlations, etc.

LaMET has opened a window for us to study the 3D hadron structures from lattice QCD. With the improvement in computational power and systematic corrections, lattice QCD will be capable of providing significant inputs for the experimental programs such as Jefferson Lab 12 GeV Upgrade and the EIC in the next decade.

\section{Acknowledgments}

This work was supported by the U.S. Department of Energy, Office of Science, Office of Nuclear Physics, from DE-SC0011090, DE-SC0012704 and within the framework of the TMD Topical Collaboration.

\section{References}

[1] A. Accardi et al., Eur. Phys. J. A 52, no. 9, 268 (2016) doi:10.1140/epja/i2016-16268-9 [arXiv:1212.1701 [nucl-ex]].

[2] X. Chen, PoS DIS 2018, 170 (2018) doi:10.22323/1.316.0170 [arXiv:1809.00448 [nucl-ex]].

[3] J. L. Abelleira Fernandez et al. [LHeC Study Group], J. Phys. G 39, 075001 (2012) doi:10.1088/0954-3899/39/7/075001 [arXiv:1206.2913 [physics.acc-ph]].

[4] H. W. Lin, W. Melnitchouk, A. Prokudin, N. Sato and H. Shows, Phys. Rev. Lett. 120, no. 15, 152502 (2018) doi:10.1103/PhysRevLett.120.152502 [arXiv:1710.09858 [hep-ph]].

[5] X. Ji, Phys. Rev. Lett. 110, 262002 (2013) doi:10.1103/PhysRevLett.110.262002 [arXiv:1305.1539 [hep-ph]].

[6] X. Ji, Sci. China Phys. Mech. Astron. 57, 1407 (2014) doi:10.1007/s11433-014-5492-3 [arXiv:1404.6680 [hep-ph]].

[7] H. W. Lin, J. W. Chen, S. D. Cohen and X. Ji, Phys. Rev. D 91, 054510 (2015) doi:10.1103/PhysRevD.91.054510 [arXiv:1402.1462 [hep-ph]].

[8] J. W. Chen, S. D. Cohen, X. Ji, H. W. Lin and J. H. Zhang, Nucl. Phys. B 911, 246 (2016) doi:10.1016/j.nuclphysb.2016.07.033 [arXiv:1603.06664 [hep-ph]].

[9] C. Alexandrou, K. Cichy, V. Drach, E. Garcia-Ramos, K. Hadjiyiannakou, K. Jansen, F. Steffens and C. Wiese, Phys. Rev. D 92, 014502 (2015) doi:10.1103/PhysRevD.92.014502 [arXiv:1504.07455 [hep-lat]]. 
[10] C. Alexandrou, K. Cichy, M. Constantinou, K. Jansen, A. Scapellato and F. Steffens, Phys. Rev. D 98, no. 9, 091503 (2018) doi:10.1103/PhysRevD.98.091503 [arXiv:1807.00232 [hep-lat]].

[11] J. W. Chen, L. Jin, H. W. Lin, Y. S. Liu, Y. B. Yang, J. H. Zhang and Y. Zhao, arXiv:1803.04393 [hep-lat].

[12] C. Alexandrou, K. Cichy, M. Constantinou, K. Jansen, A. Scapellato and F. Steffens, Phys. Rev. Lett. 121, no. 11, 112001 (2018) doi:10.1103/PhysRevLett.121.112001 [arXiv:1803.02685 [hep-lat]].

[13] H. W. Lin et al., Phys. Rev. Lett. 121, no. 24, 242003 (2018) doi:10.1103/PhysRevLett.121.242003 [arXiv:1807.07431 [hep-lat]].

[14] Y. S. Liu, J. W. Chen, L. Jin, R. Li, H. W. Lin, Y. B. Yang, J. H. Zhang and Y. Zhao, arXiv:1810.05043 [hep-lat].

[15] Z. Y. Fan, Y. B. Yang, A. Anthony, H. W. Lin and K. F. Liu, Phys. Rev. Lett. 121, no. 24, 242001 (2018) doi:10.1103/PhysRevLett.121.242001 [arXiv:1808.02077 [hep-lat]].

[16] J. W. Chen, H. W. Lin and J. H. Zhang, arXiv:1904.12376 [hep-lat].

[17] X. Ji, P. Sun, X. Xiong and F. Yuan, Phys. Rev. D 91, 074009 (2015) doi:10.1103/PhysRevD.91.074009 [arXiv:1405.7640 [hep-ph]].

[18] X. Ji, L. C. Jin, F. Yuan, J. H. Zhang and Y. Zhao, Phys. Rev. D 99, no. 11, 114006 (2019) doi:10.1103/PhysRevD.99.114006 [arXiv:1801.05930 [hep-ph]].

[19] M. A. Ebert, I. W. Stewart and Y. Zhao, Phys. Rev. D 99, no. 3, 034505 (2019) doi:10.1103/PhysRevD.99.034505 [arXiv:1811.00026 [hep-ph]].

[20] M. A. Ebert, I. W. Stewart and Y. Zhao, JHEP 1909, 037 (2019) doi:10.1007/JHEP09(2019)037 [arXiv:1901.03685 [hep-ph]].

[21] M. A. Ebert, I. W. Stewart and Y. Zhao, arXiv:1910.08569 [hep-ph].

[22] X. Ji, Y. Liu and Y. S. Liu, arXiv:1910.11415 [hep-ph].

[23] X. Ji, Y. Liu and Y. S. Liu, arXiv:1911.03840 [hep-ph].

[24] P. Shanahan, M. Wagman and Y. Zhao, arXiv:1911.00800 [hep-lat].

[25] P. Shanahan, M. Wagman and Y. Zhao, in preparation.

[26] X. Xiong, X. Ji, J. H. Zhang and Y. Zhao, Phys. Rev. D 90, no. 1, 014051 (2014) doi:10.1103/PhysRevD.90.014051 [arXiv:1310.7471 [hep-ph]].

[27] Y. Q. Ma and J. W. Qiu, Phys. Rev. D 98, no. 7, 074021 (2018) doi:10.1103/PhysRevD.98.074021 [arXiv:1404.6860 [hep-ph]].

[28] T. Izubuchi, X. Ji, L. Jin, I. W. Stewart and Y. Zhao, Phys. Rev. D 98, no. 5, 056004 (2018) doi:10.1103/PhysRevD.98.056004 [arXiv:1801.03917 [hep-ph]].

[29] J. H. Zhang, J. W. Chen, L. Jin, H. W. Lin, A. Schäfer and Y. Zhao, Phys. Rev. D 100, no. 3, 034505 (2019) doi:10.1103/PhysRevD.100.034505 [arXiv:1804.01483 [hep-lat]].

[30] T. Izubuchi, L. Jin, C. Kallidonis, N. Karthik, S. Mukherjee, P. Petreczky, C. Shugert and S. Syritsyn, Phys. Rev. D 100, no. 3, 034516 (2019) doi:10.1103/PhysRevD.100.034516 [arXiv:1905.06349 [hep-lat]].

[31] X. Ji, J. H. Zhang and Y. Zhao, Phys. Rev. Lett. 120, no. 11, 112001 (2018) doi:10.1103/PhysRevLett.120.112001 [arXiv:1706.08962 [hep-ph]]. 
[32] T. Ishikawa, Y. Q. Ma, J. W. Qiu and S. Yoshida, Phys. Rev. D 96, no. 9, 094019 (2017) doi:10.1103/PhysRevD.96.094019 [arXiv:1707.03107 [hep-ph]].

[33] J. Green, K. Jansen and F. Steffens, Phys. Rev. Lett. 121, no. 2, 022004 (2018) doi:10.1103/PhysRevLett.121.022004 [arXiv:1707.07152 [hep-lat]].

[34] B. U. Musch, P. Hagler, J. W. Negele and A. Schafer, Phys. Rev. D 83, 094507 (2011) doi:10.1103/PhysRevD.83.094507 [arXiv:1011.1213 [hep-lat]].

[35] T. Ishikawa, Y. Q. Ma, J. W. Qiu and S. Yoshida, arXiv:1609.02018 [hep-lat].

[36] J. H. Zhang, J. W. Chen, X. Ji, L. Jin and H. W. Lin, Phys. Rev. D 95, no. 9, 094514 (2017) doi:10.1103/PhysRevD.95.094514 [arXiv:1702.00008 [hep-lat]].

[37] M. Constantinou and H. Panagopoulos, Phys. Rev. D 96, no. 5, 054506 (2017) doi:10.1103/PhysRevD.96.054506 [arXiv:1705.11193 [hep-lat]].

[38] I. W. Stewart and Y. Zhao, Phys. Rev. D 97, no. 5, 054512 (2018) doi:10.1103/PhysRevD.97.054512 [arXiv:1709.04933 [hep-ph]].

[39] C. Alexandrou, K. Cichy, M. Constantinou, K. Hadjiyiannakou, K. Jansen, H. Panagopoulos and F. Steffens, Nucl. Phys. B 923, 394 (2017) doi:10.1016/j.nuclphysb.2017.08.012 [arXiv:1706.00265 [hep-lat]].

[40] J. W. Chen, T. Ishikawa, L. Jin, H. W. Lin, Y. B. Yang, J. H. Zhang and Y. Zhao, Phys. Rev. D 97, no. 1, 014505 (2018) doi:10.1103/PhysRevD.97.014505 [arXiv:1706.01295 [hep-lat]].

[41] A. V. Radyushkin, Phys. Rev. D 96, no. 3, 034025 (2017) doi:10.1103/PhysRevD.96.034025 [arXiv:1705.01488 [hep-ph]].

[42] K. Orginos, A. Radyushkin, J. Karpie and S. Zafeiropoulos, Phys. Rev. D 96, no. 9, 094503 (2017) doi:10.1103/PhysRevD.96.094503 [arXiv:1706.05373 [hep-ph]].

[43] O. Nachtmann, Nucl. Phys. B 63, 237 (1973). doi:10.1016/0550-3213(73)90144-2

[44] X. Ji and J. H. Zhang, Phys. Rev. D 92, 034006 (2015) doi:10.1103/PhysRevD.92.034006 [arXiv:1505.07699 [hep-ph]].

[45] Y. S. Liu et al., arXiv:1807.06566 [hep-lat].

[46] J. H. Zhang et al. [LP3 Collaboration], Nucl. Phys. B 939, 429 (2019) doi:10.1016/j.nuclphysb.2018.12.020 [arXiv:1712.10025 [hep-ph]].

[47] J. H. Zhang, X. Ji, A. Schäfer, W. Wang and S. Zhao, Phys. Rev. Lett. 122, no. 14, 142001 (2019) doi:10.1103/PhysRevLett.122.142001 [arXiv:1808.10824 [hep-ph]].

[48] Z. Y. Li, Y. Q. Ma and J. W. Qiu, Phys. Rev. Lett. 122, no. 6, 062002 (2019) doi:10.1103/PhysRevLett.122.062002 [arXiv:1809.01836 [hep-ph]].

[49] W. Wang, J. H. Zhang, S. Zhao and R. Zhu, Phys. Rev. D 100, no. 7, 074509 (2019) doi:10.1103/PhysRevD.100.074509 [arXiv:1904.00978 [hep-ph]].

[50] W. Wang, S. Zhao and R. Zhu, Eur. Phys. J. C 78, no. 2, 147 (2018) doi:10.1140/epjc/s10052-018-5617-3 [arXiv:1708.02458 [hep-ph]].

[51] W. Wang and S. Zhao, JHEP 1805, 142 (2018) doi:10.1007/JHEP05(2018)142 [arXiv:1712.09247 [hep-ph]].

[52] M. Burkardt, Phys. Rev. D 62, 071503 (2000) Erratum: [Phys. Rev. D 66, 119903 (2002)] doi:10.1103/PhysRevD.62.071503, 10.1103/PhysRevD.66.119903 [hep-ph/0005108]. 
[53] M. Burkardt, Int. J. Mod. Phys. A 18, 173 (2003) doi:10.1142/S0217751X03012370 [hep-ph/0207047].

[54] Y. S. Liu, W. Wang, J. Xu, Q. A. Zhang, J. H. Zhang, S. Zhao and Y. Zhao, Phys. Rev. D 100, no. 3, 034006 (2019) doi:10.1103/PhysRevD.100.034006 [arXiv:1902.00307 [hep-ph]].

[55] J. C. Collins and D. E. Soper, Nucl. Phys. B 193, 381 (1981) Erratum: [Nucl. Phys. B 213, 545 (1983)]. doi:10.1016/0550-3213(81)90339-4

[56] J. C. Collins and D. E. Soper, Nucl. Phys. B 197, 446 (1982). doi:10.1016/0550-3213(82)90453-9

[57] J. C. Collins, D. E. Soper and G. F. Sterman, Nucl. Phys. B 250, 199 (1985). doi:10.1016/0550-3213(85)90479-1

[58] T. Becher and M. Neubert, Eur. Phys. J. C 71, 1665 (2011) doi:10.1140/epjc/s10052-011-1665-7 [arXiv:1007.4005 [hep-ph]].

[59] T. Becher, M. Neubert and D. Wilhelm, JHEP 1202, 124 (2012) doi:10.1007/JHEP02(2012)124 [arXiv:1109.6027 [hep-ph]].

[60] T. Becher, M. Neubert and D. Wilhelm, JHEP 1305, 110 (2013) doi:10.1007/JHEP05(2013)110 [arXiv:1212.2621 [hep-ph]].

[61] M. G. Echevarria, A. Idilbi and I. Scimemi, JHEP 1207, 002 (2012) doi:10.1007/JHEP07(2012)002 [arXiv:1111.4996 [hep-ph]].

[62] M. G. Echevarría, A. Idilbi and I. Scimemi, Phys. Lett. B 726, 795 (2013) doi:10.1016/j.physletb.2013.09.003 [arXiv:1211.1947 [hep-ph]].

[63] M. G. Echevarria, A. Idilbi and I. Scimemi, Phys. Rev. D 90, no. 1, 014003 (2014) doi:10.1103/PhysRevD.90.014003 [arXiv:1402.0869 [hep-ph]].

[64] J. Y. Chiu, A. Jain, D. Neill and I. Z. Rothstein, JHEP 1205, 084 (2012) doi:10.1007/JHEP05(2012)084 [arXiv:1202.0814 [hep-ph]].

[65] J. Collins, Camb. Monogr. Part. Phys. Nucl. Phys. Cosmol. 32, 1 (2011).

[66] P. Hagler, B. U. Musch, J. W. Negele and A. Schafer, EPL 88, no. 6, 61001 (2009) doi:10.1209/0295-5075/88/61001 [arXiv:0908.1283 [hep-lat]].

[67] B. U. Musch, P. Hagler, M. Engelhardt, J. W. Negele and A. Schafer, Phys. Rev. D 85, 094510 (2012) doi:10.1103/PhysRevD.85.094510 [arXiv:1111.4249 [hep-lat]].

[68] M. Engelhardt, P. Hägler, B. Musch, J. Negele and A. Schäfer, Phys. Rev. D 93, no. 5, 054501 (2016) doi:10.1103/PhysRevD.93.054501 [arXiv:1506.07826 [hep-lat]].

[69] B. Yoon et al., Proceedings of Science LATTICE 2015 (2016) 116 [arXiv:1601.05717 [hep-lat]].

[70] B. Yoon et al., Phys. Rev. D 96, no. 9, 094508 (2017) doi:10.1103/PhysRevD.96.094508 [arXiv:1706.03406 [hep-lat]].

[71] M. Constantinou, H. Panagopoulos and G. Spanoudes, Phys. Rev. D 99, no. 7, 074508 (2019) doi:10.1103/PhysRevD.99.074508 [arXiv:1901.03862 [hep-lat]]. 